\section{Revisiting induction chemotherapy before radiotherapy for head and neck cancer, part II: nasopharyngeal carcinoma}

\author{
"Delaying concurrent chemoradiation for the purposes of initiating \\ induction chemotherapy should generally be reserved for situations of \\ practical or logistical barriers to chemoradiation or as a defined \\ operational procedure, preferably as part of a clinical trial."
}

\section{Christopher H Chapman', Upendra Parvathaneni ${ }^{2}$ \& Sue S Yom ${ }^{*, 1}$}

First draft submitted: 11 December 2016; Accepted for publication: 6 January 2017; Published online: 7 February 2017

\section{Background of induction chemotherapy for nasopharyngeal carcinoma}

We have separately outlined the history and evidence basis of induction chemotherapy for non-nasopharyngeal head and neck carcinomas, describing results for the anatomic subsites tested in western countries: oral cavity, oropharynx, larynx and hypopharynx [1]. On the other hand, nasopharyngeal carcinoma, which is rare in Europe and North America, is much more common in southeast Asia, the Middle East and among the Inuit populations. The nonkeratinizing and undifferentiated endemic types are highly associated with Epstein-Barr virus (EBV). The clinical behavior of endemic nasopharyngeal carcinoma is also distinct, affecting younger patients and having a high propensity to nodal and distant metastasis [2]. For these reasons, nasopharyngeal carcinoma has historically been addressed in separate clinical trials and a different staging system has been developed. Yet despite these differences, the history of induction chemotherapy in nasopharyngeal carcinoma has paralleled that in other sites, with generations of trials investigating cisplatin/5FU $(\mathrm{PF})$, the addition of taxanes (TPF), and the use of agents targeting the EGFR.

\section{The establishment of adjuvant} chemotherapy

In 1998, the North American Intergroup 0099 trial showed that concurrent cisplatin-radiotherapy prolonged survival compared with radiation alone [3], a conclusion that was confirmed by a subsequent trial in the endemic population [4]. Importantly, both of these trials also included an extended course of adjuvant cisplatin/5FU. Compliance with the adjuvant chemotherapy phase was low in both trials, leaving some uncertainty about its relative importance. Similar to the MACH-NC analysis in other sites, the MAC-NPC meta-analysis examined the effect of timing of chemotherapy on results for nasopharyngeal carcinoma $[5,6]$. This study only identified an overall survival benefit for concurrent chemotherapy versus radiation alone, although locoregional and distant control were both improved

\section{KEYWORDS}

- antineoplastic agents

- antineoplastic combined chemotherapy protocols cell - carcinoma • combined modality therapy • induction therapy

- molecular targeted therapy

- nasopharyngeal neoplasms

- neoadjuvant therapy • sequential therapy • squamous • treatment outcomes

“...nasopharyngeal carcinoma has historically been addressed in separate clinical trials and a different staging system has been developed." 
"...the addition of adjuvant chemotherapy to concurrent chemoradiotherapy for nasopharyngeal carcinoma has persisted as a part of the standard of care ... although the evidence for its use has been mixed." with induction chemotherapy versus radiation alone.

The updated meta-analysis in 2015 attempted to compare concurrent plus adjuvant chemotherapy to concurrent chemotherapy alone. A significant overall survival benefit was seen for concurrent chemotherapy $(+5.3 \%$ at 5 years, as compared with radiation alone), and a greater magnitude of benefit was seen for concurrent plus adjuvant $(+12.4 \%$ at 5 years), but there was no statistically significant difference between the two groups. Although there were questions about the heterogeneity of the studies included in the meta-analysis, these results at least suggested that adding more chemotherapy could potentially improve survival outcomes. In contrast to other head and neck sites, because of the relatively low rates of locoregional failure and high rates of distant failure seen in nasopharyngeal cancer, this hypothesis appeared to be biologically plausible. However, there was no comparison of concurrent with adjuvant versus concurrent with induction in this meta-analysis, so the potential advantage of induction as opposed to the standard adjuvant phase remained unclear.

In the Intergroup 0099 study, only $55 \%$ of patients were able to complete adjuvant chemotherapy. However, a post-hoc analysis of similar studies found an association between completion of adjuvant chemotherapy and freedom from distant and locoregional recurrence [7]. It was hypothesized that switching from adjuvant to induction chemotherapy would improve outcomes by allowing for more completion of chemotherapy before radiation, at a time when cumulative toxicity would be lower. This was directly tested by NPC-0501, a multiarm study in which patients were randomized to either adjuvant or induction chemotherapy (using either cisplatin/5FU or cisplatin/capecitabine) in addition to concurrent cisplatin-radiotherapy [8] This study resulted in $88 \%$ of induction patients completing all chemotherapy, versus only $64 \%$ of adjuvant-assigned patients. However, although a multivariate analysis found improved progression-free and overall survival associated with the induction arms, the unadjusted comparisons were nonsignificant and findings were considered inconclusive in the end.

A new meta-analysis in publication has further examined chemotherapy sequencing, and like MAC-NPC finds the greatest survival advantage for concurrent plus adjuvant, but not significantly different than concurrent alone [9].
Induction plus concurrent was superior to radiation alone, and not statistically different than concurrent alone or adjuvant plus concurrent for overall survival. Intriguingly, induction plus concurrent had the greatest benefit for distant control, suggesting a path for future investigation in subgroups at high metastatic risk.

\section{Taxane-based induction for nasopharyngeal carcinoma}

As in other sites, the arrival of taxanes revived interest in induction for nasopharyngeal carcinoma, especially after a randomized Phase II trial found a $26 \%$ absolute improvement in 3 -year overall survival with induction TPF versus concurrent cisplatin radiotherapy alone [10]. However, results from further studies with taxanes were mixed. A Phase II study from Greece found no difference in overall response rate or other end points when adding cisplatin/epirubicin/paclitaxel induction to concurrent cisplatin radiotherapy [11]. Another Phase II/III study from Singapore found no benefit from the addition of gemcitabine/carboplatin/paclitaxel induction [12]. Yet, a recently published Phase III trial testing the addition of TPF induction did find significant improvements in overall survival and distant-failure-free survival in the induction arm [13]. The reasons for the discrepancies in these various trial results are unclear, but may result from differences in patient populations, statistical power, and treatment regimens. Another area of uncertainty is the lack of stratification by circulating EBV DNA levels, a prognostic factor in nasopharyngeal carcinoma [14]. In the aggregate, these trials suggest that induction TPF in particular could improve outcomes compared with chemoradiotherapy alone, although the comparison of induction versus adjuvant chemotherapy remains unclear. In contrast to other head and neck sites, there seems to be more possibility that the additional toxicity from induction chemotherapy might be balanced by improvements in survival in nasopharyngeal carcinoma.

\section{Induction to select for reduced-intensity radiation-based treatment}

Like in other sites, EGFR-targeted agents have been investigated as a way of lowering toxicity while accruing the benefits of additional therapy. A recently presented multi-institutional study treated patients with locally advanced nasopharyngeal carcinoma with induction TPF, and then randomized the patients to either concurrent 
cisplatin or the EGFR-antibody nimotuzumab with radiation (NCT02012062) [15]. The primary end point of the study was toxicity. Treatmentrelated cytopenias, anorexia, nausea and fatigue were all less frequent with nimotuzumab, and the completion rate was $97 \&$ versus $40 \%$ for cisplatin. Like the French TREMPLIN trial for laryngeal carcinoma and the Spanish Head and Neck Cancer Group 2007-01 trial for other sites, no differences were seen in rates of overall or progression-free survival. If these results are validated for survival end points, presumably patients could safely receive a more tolerable EGFR-based concurrent regimen following induction.

An alternative strategy being investigated by the multi-institutional NRG-HN001 study (NCT02135042) uses the postchemoradiation plasma EBV DNA level as a biomarker of response, which may be more predictive than the pretreatment level [16]. After concurrent chemoradiation, this trial assigns patients to adjuvant chemotherapy by their risk level as determined by detectability of EBV DNA, with the twin goals of shifting the intensity (and toxicity) of additional treatment to those who might benefit most from it, while testing the safety of eliminating chemotherapy for those in whom EBV DNA becomes undetectable. Patients who have detectable EBV DNA are randomized to cisplatin/5FU or an experimental regimen of gemcitabine/paclitaxel. Patients who have no detectable EBV DNA are randomized to the current standard of care, consisting of adjuvant cisplatin $/ 5 \mathrm{FU}$, or no further treatment. The EBV DNA assay used in this study was harmonized among several participating test sites so that the results are consistent among participating centers [17].

\section{Conclusion}

In summary, the addition of adjuvant chemotherapy to concurrent chemoradiotherapy for nasopharyngeal carcinoma has persisted as a part of the standard of care since its use in the original Intergroup 0099 protocol, although the evidence for its use has been mixed. While additional chemotherapy generally appears to be of value in the treatment of this disease, the optimal timing, agents and population are still to be determined. It is not clear whether induction chemotherapy with newer regimens such as TPF may ultimately be superior to adjuvant chemotherapy, and the introduction of EGFRtargeted agents has created promising new combinations. However, the data from randomized trials are still maturing. At present, like in other head and neck sites, the cornerstone of therapy is concurrent chemoradiation. Delaying concurrent chemoradiation for the purposes of initiating induction chemotherapy should generally be reserved for situations of practical or logistical barriers to chemoradiation or as a defined operational procedure, preferably as part of a clinical trial. Risk stratification with potentially predictive molecular markers such as circulating EBV DNA may further help select patients for the most appropriate treatment.

\section{Financial \& competing interests disclosure}

SS Yom received a research grant from Genentech, honorarium from Astra-Zeneca, and royalty from $U_{p} T o D a t e$. The authors have no other relevant affiliations or financial involvement with any organization or entity with a financial interest in or financial conflict with the subject matter or materials discussed in the manuscript apart from those disclosed.

No writing assistance was utilized in the production of this manuscript.

\section{Open access}

This work is licensed under the Creative Commons Attribution-NonCommercial 4.0 Unported License. To view a copy of this license, visit http://creativecommons.org/ licenses/by-nc-nd/4.0/

\section{References}

1 Chapman CH, Parvathaneni U, Yom SS. Revisiting induction chemotherapy before radiotherapy for head and neck cancer, part I: carcinoma of non-nasopharyngeal sites. Future Oncol. 13(6), 469-475 (2017).

2 Altun M, Fandi A, Dupuis O, Cvitkovic E, Krajina Z, Eschwege F. Undifferentiated nasopharyngeal cancer (UCNT): current diagnostic and therapeutic aspects. Int. J.
Radiat. Oncol. Biol. Phys. 32(3), 859-877 (1995).

3 Al-Sarraf M, LeBlanc M, Giri PG et al. Chemoradiotherapy versus radiotherapy in patients with advanced nasopharyngeal cancer: Phase III randomized Intergroup study 0099. J Clin Oncol. 16(4), 1310-1317 (1998).

4 Wee J, Tan EH, Tai BC et al. Randomized trial of radiotherapy versus concurrent chemoradiotherapy followed by adjuvant chemotherapy in patients with American Joint Committee on Cancer/International Union against cancer stage III and IV nasopharyngeal cancer of the endemic variety. J. Clin. Oncol. 23(27), 6730-6738 (2005).

5 Baujat B, Audry H, Bourhis J et al. Chemotherapy in locally advanced nasopharyngeal carcinoma: an individual patient data meta-analysis of eight 
randomized trials and 1753 patients. Int. J. Radiat. Oncol. Biol. Phys. 64(1), 47-56 (2006).

Blanchard P, Lee A, Marguet S et al. Chemotherapy and radiotherapy in nasopharyngeal carcinoma: an update of the MAC-NPC meta-analysis. Lancet Oncol. 16(6), 645-655 (2015).

7 Lee AWM, Tung SY, Ngan RKC et al. Factors contributing to the efficacy of concurrentadjuvant chemotherapy for locoregionally advanced nasopharyngeal carcinoma: combined analyses of NPC-9901 and NPC-9902 Trials. Eur. J. Cancer 47(5), 656-666 (2011).

8 Lee AWM, Ngan RKC, Tung SY et al. Preliminary results of trial NPC-0501 evaluating the therapeutic gain by changing from concurrent-adjuvant to inductionconcurrent chemoradiotherapy, changing from fluorouracil to capecitabine, and changing from conventional to accelerated radiotherapy fractionation in patients with locoregionally advanced nasopharyngeal carcinoma. Cancer 121(8), 1328-1338 (2015).

9 Ribassin-Majed L, Marguet S, Lee AW et al. What is the best treatment of locally advanced nasopharyngeal carcinoma? An individual patient data network meta-analysis. J. Clin. Oncol. 5, JCO2016674119 (2016).

10 Hui EP, Ma BB, Leung SF et al. Randomized Phase II trial of concurrent cisplatinradiotherapy with or without neoadjuvant docetaxel and cisplatin in advanced nasopharyngeal carcinoma. J. Clin. Oncol. 27(2), 242-249 (2009).

11 Fountzilas G, Ciuleanu E, Bobos M et al. Induction chemotherapy followed by concomitant radiotherapy and weekly cisplatin versus the same concomitant chemoradiotherapy in patients with nasopharyngeal carcinoma: a randomized Phase II study conducted by the Hellenic Cooperative Oncology Group (HeCOG) with biomarker evaluation. Ann. Oncol. 23(2), 427-435 (2012).

12 Tan T, Lim WT, Fong K-W et al. Concurrent chemo-radiation with or without induction gemcitabine, Carboplatin, and Paclitaxel: a randomized, Phase $2 / 3$ trial in locally advanced nasopharyngeal carcinoma. Int. J. Radiat. Oncol. Biol. Phys. 91(5), 952-960 (2015).

13 Sun Y, Li W-F, Chen N-Y et al. Induction chemotherapy plus concurrent chemoradiotherapy versus concurrent chemoradiotherapy alone in locoregionally advanced nasopharyngeal carcinoma: a Phase 3, multicentre, randomised controlled trial. Lancet Oncol. 17(11), 1509-1520 (2016).

14 Lo YM, Chan AT, Chan LY et al. Molecular prognostication of nasopharyngeal carcinoma by quantitative analysis of circulating EpsteinBarr virus DNA. Cancer Res. 60(24), 6878-6881 (2000).

15 Kong L, Lin Q, Hu C et al. Radiation plus concurrent nimotuzumab versus CDDP in locally advanced nasopharyngeal cancer: results of a Phase III randomised trial. J. Clin. Oncol. (34), abst 6002 (2016).

16 Lin J-C, Wang W-Y, Chen KY et al. Quantification of plasma Epstein-Barr virus DNA in patients with advanced nasopharyngeal carcinoma. N. Engl. J. Med. 350(24), 2461-2470 (2004).

17 Le QT, Zhang Q, Cao H et al. An international collaboration to harmonize the quantitative plasma Epstein-Barr virus DNA assay for future biomarker-guided trials in nasopharyngeal carcinoma. Clin. Cancer Res. 19(8), 2208-2215 (2013). 